Series representations of the remainders in the expansions for certain functions with applications

Chen, Chao-Ping

Paris, Richard B.

The final publication is available at Springer via:

http://dx.doi.org/10.1007/s00025-016-0612-1 


\title{
SERIES REPRESENTATIONS OF THE REMAINDERS IN THE EXPANSIONS FOR CERTAIN FUNCTIONS WITH APPLICATIONS
}

\author{
CHAO-PING CHEN* AND RICHARD B. PARIS
}

\begin{abstract}
We present a summary of the series representations of the remainders in the expansions in ascending powers of $t$ of $2 /\left(e^{t}+1\right)$, sech $t$ and $\operatorname{coth} t$ and establish simple bounds for these remainders when $t>0$. Several applications of these expansions are given which enable us to deduce some inequalities and completely monotonic functions associated with the ratio of two gamma functions. In addition, we derive a (presumably new) quadratic recurrence relation for the Bernoulli numbers $B_{n}$.
\end{abstract}

\section{Introduction}

The Bernoulli polynomials $B_{n}(x)$ and Euler polynomials $E_{n}(x)$ are defined, respectively, by the generating functions:

$$
\frac{t e^{x t}}{e^{t}-1}=\sum_{n=0}^{\infty} B_{n}(x) \frac{t^{n}}{n !} \quad(|t|<2 \pi) \quad \text { and } \quad \frac{2 e^{x t}}{e^{t}+1}=\sum_{n=0}^{\infty} E_{n}(x) \frac{t^{n}}{n !} \quad(|t|<\pi) .
$$

The numbers $B_{n}=B_{n}(0)$ and $E_{n}=2^{n} E_{n}\left(\frac{1}{2}\right)$, which are known to be rational numbers and integers, respectively, are called Bernoulli and Euler numbers.

It follows from [25, Chapter 4, Part I, Problem 154] that

$$
\sum_{j=1}^{2 m} \frac{B_{2 j}}{(2 j) !} t^{2 j}<\frac{t}{e^{t}-1}-1+\frac{t}{2}<\sum_{j=1}^{2 m+1} \frac{B_{2 j}}{(2 j) !} t^{2 j}
$$

for $t>0$ and $m \in \mathbb{N}_{0}:=\mathbb{N} \cup\{0\}, \mathbb{N}:=\{1,2,3, \ldots\}$. The inequality (1.1) can be also found in $[12,26]$. It is also known [33, p. 64] that for integer $m \geq 2$,

$$
\frac{t}{e^{t}-1}-1+\frac{t}{2}=\sum_{j=1}^{m-1} \frac{B_{2 j}}{(2 j) !} t^{2 j}+(-1)^{m-1} t^{2 m} s_{m}(t),
$$

where

$$
s_{m}(t)=\sum_{k=1}^{\infty} \frac{2}{(2 k \pi)^{2 m-2}\left(t^{2}+(2 k \pi)^{2}\right)} .
$$

It is easily seen that (1.2) implies (1.1).

Binet's first formula [32, p. 16] for the logarithm of $\Gamma(x)$ states that

$$
\ln \Gamma(x)=\left(x-\frac{1}{2}\right) \ln x-x+\ln \sqrt{2 \pi}+\int_{0}^{\infty}\left(\frac{t}{e^{t}-1}-1+\frac{t}{2}\right) \frac{e^{-x t}}{t^{2}} \mathrm{~d} t \quad(x>0) .
$$

*Corresponding Author.

2010 Mathematics Subject Classification. Primary 11B68; Secondary 25A48, 26 D15.

Key words and phrases. Bernoulli polynomials and numbers; Euler polynomials and numbers; Completely monotonic functions. 
Combining (1.2) with (1.3), $\mathrm{Xu}$ and Han [38] deduced in 2009 that for every $m \in \mathbb{N}_{0}$, the function

$$
R_{m}(x)=(-1)^{m}\left[\ln \Gamma(x)-\left(x-\frac{1}{2}\right) \ln x+x-\ln \sqrt{2 \pi}-\sum_{j=1}^{m} \frac{B_{2 j}}{2 j(2 j-1) x^{2 j-1}}\right]
$$

is completely monotonic on $(0, \infty)$. Recall that a function $f(x)$ is said to be completely monotonic on an interval $I$ if it has derivatives of all orders on $I$ and satisfies the following inequality:

$$
(-1)^{n} f^{(n)}(x) \geq 0 \quad\left(x \in I, \quad n \in \mathbb{N}_{0}\right) .
$$

For $m=0$, the complete monotonicity property of $R_{m}(x)$ was proved by Muldoon [21]. Alzer [2] first proved in 1997 that $R_{m}(x)$ is completely monotonic on $(0, \infty)$. In 2006, Koumandos [12] proved the double inequality (1.1), and then used (1.1) and (1.3) to give a simpler proof of the complete monotonicity property of $R_{m}(x)$. In 2009, Koumandos and Pedersen [13, Theorem 2.1] strengthened this result.

Chen and Paris [9, Lemma 1] presented an analogous result to (1.1) given by

$$
\sum_{j=2}^{2 m+1} \frac{\left(1-2^{2 j}\right) B_{2 j}}{j} \frac{t^{2 j-1}}{(2 j-1) !}<\frac{2}{e^{t}+1}-1+\frac{t}{2}<\sum_{j=2}^{2 m} \frac{\left(1-2^{2 j}\right) B_{2 j}}{j} \frac{t^{2 j-1}}{(2 j-1) !}
$$

for $t>0$ and $m \in \mathbb{N}$. This inequality can also be written for $t>0$ and $m \in \mathbb{N}_{0}$ as

$$
(-1)^{m+1}\left(\frac{2}{e^{t}+1}-1-\sum_{j=1}^{m} \frac{\left(1-2^{2 j}\right) B_{2 j}}{j} \frac{t^{2 j-1}}{(2 j-1) !}\right)>0 .
$$

Based on the inequality (1.7), Chen and Paris [9, Theorem 1] proved that for every $m \in \mathbb{N}_{0}$, the function

$$
F_{m}(x)=(-1)^{m}\left[\ln \left(\frac{\Gamma(x+1)}{\Gamma\left(x+\frac{1}{2}\right)}\right)-\frac{1}{2} \ln x-\sum_{j=1}^{m}\left(1-\frac{1}{2^{2 j}}\right) \frac{B_{2 j}}{j(2 j-1) x^{2 j-1}}\right]
$$

is completely monotonic on $(0, \infty)$. This result is similar to the complete monotonicity property of $R_{m}(x)$ in (1.4). In analogy with (1.2), these authors also considered [9, Eq. (2.4)] the remainder in the truncated expansion in ascending powers of $t$ of the function $2 /\left(e^{t}+1\right)$ and gave an integral representation for this remainder when $t>0$. Similar expansions for $2 /\left(e^{t}+1\right)$ and sech $t$ have recently been obtained by Koumandos in [15, Section 4].

In this paper, we summarize the series representations of the remainders in the expansions of $2 /\left(e^{t}+1\right), \operatorname{coth} t$ and $\operatorname{sech} t$ and establish simple bounds for these remainders when $t>0$. We also obtain the double inequality for $t>0$ and $m \in \mathbb{N}_{0}$,

$$
\sum_{j=0}^{2 m+1} \frac{E_{2 j}}{(2 j) !} t^{2 j}<\operatorname{sech} t<\sum_{j=0}^{2 m} \frac{E_{2 j}}{(2 j) !} t^{2 j} .
$$

Several applications of these results are presented in Section 3, where some complete monotonicity results and a double inequality for a ratio of factorials are considered. Finally, in Section 4, we derive a quadratic recurrence relation for the Bernoulli numbers given in Theorem 4.1.

\section{Main results}

In this section we summarize the expansions of three hyperbolic functions given by the following theorem: 
Theorem 2.1. For $t>0$ and integer $m \geq 1$, we have the expansions

$$
\begin{aligned}
\frac{2}{e^{t}+1} & =1+\sum_{j=1}^{m-1} \frac{2\left(1-2^{2 j}\right) B_{2 j}}{(2 j) !} t^{2 j-1}+(-1)^{m} t^{2 m-1} p_{m}(t), \\
\operatorname{coth} t & =\sum_{j=0}^{m-1} \frac{2^{2 j} B_{2 j}}{(2 j) !} t^{2 j-1}+(-1)^{m-1} t^{2 m-1} q_{m}(t), \\
\frac{1}{\cosh t} & =\sum_{j=0}^{m-1} \frac{E_{2 j}}{(2 j) !} t^{2 j}+(-1)^{m} t^{2 m} r_{m}(t),
\end{aligned}
$$

where the remainder functions $p_{m}(t), q_{m}(t)$ and $r_{m}(t)$ are defined by

$$
\begin{aligned}
& p_{m}(t)=\sum_{k=0}^{\infty} \frac{4}{[(2 k+1) \pi]^{2 m-2}\left(t^{2}+[(2 k+1) \pi]^{2}\right)}, \\
& q_{m}(t)=\sum_{k=1}^{\infty} \frac{2}{(k \pi)^{2 m-2}\left(t^{2}+(k \pi)^{2}\right)}, \quad r_{m}(t)=\sum_{k=0}^{\infty} \frac{2(-1)^{k}}{\left[\left(k+\frac{1}{2}\right) \pi\right]^{2 m-1}\left(t^{2}+\left[\left(k+\frac{1}{2}\right) \pi\right]^{2}\right)} .
\end{aligned}
$$

Proofs of the expansions in (2.1) and (2.3) are given in [15, Section 4]. The expansion (2.2) follows in a straightforward manner from (1.2) upon noting that

$$
\operatorname{coth} t=\frac{e^{t}+e^{-t}}{e^{t}-e^{-t}}=1+\frac{2}{e^{2 t}-1}
$$

and making the change of variable $t \rightarrow 2 t$.

Corollary 2.1. For $t>0$ and integer $m \geq 1$, the expansions in Theorem 2.1 can be expressed in the form

$$
\begin{aligned}
& \frac{2}{e^{t}+1}=1+\sum_{j=1}^{m-1} \frac{2\left(1-2^{2 j}\right) B_{2 j}}{(2 j) !} t^{2 j-1}+\frac{2\left(1-2^{2 m}\right) B_{2 m}}{(2 m) !} t^{2 m-1} \Theta_{1}(t, m), \\
& \operatorname{coth} t=\sum_{j=0}^{m-1} \frac{2^{2 j} B_{2 j}}{(2 j) !} t^{2 j-1}+\frac{2^{2 m} B_{2 m}}{(2 m) !} t^{2 m-1} \Theta_{2}(t, m), \\
& \frac{1}{\cosh t}=\sum_{j=0}^{m-1} \frac{E_{2 j}}{(2 j) !} t^{2 j}+\frac{E_{2 m}}{(2 m) !} t^{2 m} \Theta_{3}(t, m),
\end{aligned}
$$

where $0<\Theta_{r}(t, m)<1$ for $r=1,2,3$.

Proof. To show (2.6) we express the remainder $p_{m}(t)$ in $(2.4)$ as

$$
p_{m}(t)=\frac{4 \Theta_{1}(t, m)}{\pi^{2 m}} \sum_{k=0}^{\infty} \frac{1}{(2 k+1)^{2 m}}, \quad \Theta_{1}(t, m)=\frac{F_{1}(t)}{F_{1}(0)},
$$

where

$$
F_{1}(t):=\sum_{k=0}^{\infty} \frac{1}{(2 k+1)^{2 m-2}\left(t^{2}+[(2 k+1) \pi]^{2}\right)} .
$$


Since

$$
\sum_{k=0}^{\infty} \frac{1}{(2 k+1)^{2 m}}=\left(1-2^{-2 m}\right) \zeta(2 m)=\left(1-2^{-2 m}\right) \frac{(2 \pi)^{2 m}}{2(2 m) !}\left|B_{2 m}\right|,
$$

we find that

$$
p_{m}(t)=\frac{2\left(2^{2 m}-1\right)}{(2 m) !}\left|B_{2 m}\right| \Theta_{1}(t, m)=(-1)^{m} \frac{2\left(1-2^{2 m}\right) B_{2 m}}{(2 m) !} \Theta_{1}(t, m)
$$

upon use of the fact that $B_{2 m}=(-1)^{m-1}\left|B_{2 m}\right|$. It is clear that $F_{1}(t)>0$ and is a decreasing function on $[0, \infty)$. Hence, for all $t>0$ and integer $m \geq 1$, we have $0<F_{1}(t)<F_{1}(0)$ and thus $0<\Theta_{1}(t, m)<1$, thereby establishing $(2.6)$.

For (2.7), we write the remainder $q_{m}(t)$ in $(2.5)$ as

$$
q_{m}(t)=\frac{2 \Theta_{2}(t, m)}{\pi^{2 m}} \sum_{k=1}^{\infty} \frac{1}{k^{2 m}}, \quad \Theta_{2}(t, m)=\frac{F_{2}(t)}{F_{2}(0)},
$$

where

$$
F_{2}(t):=\sum_{k=1}^{\infty} \frac{1}{k^{2 m-2}\left(t^{2}+(k \pi)^{2}\right)} .
$$

It is well known that

$$
\sum_{k=1}^{\infty} \frac{1}{k^{2 m}}=\frac{(-1)^{m-1}(2 \pi)^{2 m} B_{2 m}}{2(2 m) !}
$$

and hence

$$
q_{m}(t)=\frac{2^{2 m} B_{2 m}}{(2 m) !} t^{2 m-1} \Theta_{2}(t, m) .
$$

By the same reasoning as above it follows that $0<F_{2}(t)<F_{2}(0)$ and thus, for all $t>0$ and integer $m \geq 1$, we have $0<\Theta_{2}(t, m)<1$, thereby establishing $(2.7)$.

Finally, the remainder $r_{m}(t)$ in $(2.5)$ can be written as

$$
r_{m}(t)=\frac{2 \Theta_{3}(t, m)}{\left(\frac{1}{2} \pi\right)^{2 m+1}} \sum_{k=0}^{\infty} \frac{(-1)^{k}}{(2 k+1)^{2 m+1}}, \quad \Theta_{3}(t, m)=\frac{F_{3}(t)}{F_{3}(0)},
$$

where where

$$
F_{3}(t):=\sum_{k=0}^{\infty}(-1)^{k} A_{k}, \quad A_{k}:=\frac{1}{(2 k+1)^{2 m-1}\left(t^{2}+[(2 k+1) \pi / 2]^{2}\right)} .
$$

From [1, p. 807], we have

$$
\sum_{k=0}^{\infty} \frac{(-1)^{k}}{(2 k+1)^{2 m+1}}=\frac{(-1)^{m} \pi^{2 m+1}}{2^{2 m+2}(2 m) !} E_{2 m}
$$

and hence

$$
r_{m}(t)=\frac{(-1)^{m} E_{2 m}}{(2 m) !} \Theta_{3}(t, m) .
$$

Then it is easily seen that $A_{2 k}>A_{2 k+1}$ for $k \in N_{0}$ and integer $m \geq 1$; thus $F_{3}(t)>0$ for $t>0$. Differentiation yields

$$
F_{3}^{\prime}(t)=-2 t \sum_{k=0}^{\infty} \frac{(-1)^{k} A_{k}}{t^{2}+[(2 k+1) \pi / 2]^{2}}
$$


and a similar reasoning shows that $F_{3}^{\prime}(t)<0$ for $t>0$. Hence, for all $t>0$ and integer $m \geq 1$, we have $0<F_{3}(t)<F_{3}(0)$ and thus $0<\Theta_{3}(t, m)<1$. The proof of Corollary 2.1 is complete.

Remark 2.1. From (2.1) we retrieve (1.7).

Corollary 2.2. For $t>0$ and $m \in \mathbb{N}$, we have

$$
(-1)^{m}\left(\frac{2 e^{t}}{\left(e^{t}+1\right)^{2}}-\sum_{j=1}^{m} \frac{\left(2^{2 j}-1\right) B_{2 j}}{j \cdot(2 j-2) !} t^{2 j-2}\right)>0 .
$$

Proof. Differentiating the expression in (2.1) with $m$ replaced by $m+1$, we find

$$
-\frac{2 e^{t}}{\left(e^{t}+1\right)^{2}}=-\sum_{j=1}^{m} \frac{\left(2^{2 j}-1\right) B_{2 j}}{j \cdot(2 j-2) !} t^{2 j-2}+(-1)^{m+1}\left(t^{2 m+1} p_{m+1}(t)\right)^{\prime} .
$$

It is easy to see that

$$
t^{2} p_{m+1}(t)+p_{m}(t)=\frac{4}{\pi^{2 m}} \sum_{k=0}^{\infty} \frac{1}{(2 k+1)^{2 m}}=\frac{4}{\pi^{2 m}}\left(1-2^{-2 m}\right) \zeta(2 m),
$$

where $\zeta(z)$ is the Riemann zeta function. This last expression can be written as

$$
t^{2} p_{m+1}(t)=\frac{4}{\pi^{2 m}}\left(1-2^{-2 m}\right) \zeta(2 m)-p_{m}(t) .
$$

Then, since $p_{m}(t)$ is strictly decreasing for $t>0$, we deduce from (2.10) that $t^{2} p_{m+1}(t)$ is strictly increasing for $t>0$. Hence, $t^{2 m+1} p_{m+1}(t)$ is strictly increasing for $t>0$, and we then obtain

$$
(-1)^{m}\left(\frac{2 e^{t}}{\left(e^{t}+1\right)^{2}}-\sum_{j=1}^{m} \frac{\left(2^{2 j}-1\right) B_{2 j}}{j \cdot(2 j-2) !} t^{2 j-2}\right)=\left(t^{2 m+1} p_{m+1}(t)\right)^{\prime}>0
$$

for $t>0$ and $m \in \mathbb{N}$. The proof is complete.

Remark 2.2. From [22, p. 592, Eq. (24.7.9)] and [34, p. 43, Ex. 12(i)] we have

$$
E_{2 n}(x)=(-1)^{n} \sin (\pi x) \int_{0}^{\infty} \frac{4 t^{2 n} \cosh (\pi t)}{\cosh (2 \pi t)-\cos (2 \pi x)} \mathrm{d} t \quad\left(0<x<1, \quad n \in \mathbb{N}_{0}\right),
$$

from which it follows that

$$
E_{4 m}(x)>0 \quad \text { and } \quad E_{4 m+2}(x)<0 \quad\left(0<x<1, \quad m \in \mathbb{N}_{0}\right) .
$$

Use of this result enables us to deduce (1.9) from (2.8). Note that the inequality (1.9) can also be written as

$$
(-1)^{m+1}\left(\operatorname{sech} t-\sum_{j=0}^{m} \frac{E_{2 j}}{(2 j) !} t^{2 j}\right)>0 \quad\left(t>0, m \in \mathbb{N}_{0}\right) .
$$

\section{Miscellaneous results}

In this section we present several results that can be deduced from the expansions given in Section 2 . 


\subsection{The proof of a conjecture of Chen.}

In [6], Chen proposed the following conjecture.

Conjecture 3.1.. For $t>0$ and $m \in \mathbb{N}_{0}$, let

$$
\nu_{m}(t)=\frac{e^{t / 4}-e^{3 t / 4}}{e^{t}-1}-\sum_{j=0}^{m} \frac{2 B_{2 j+1}\left(\frac{1}{4}\right)}{(2 j+1) !} t^{2 j},
$$

where $B_{n}(x)$ denotes the Bernoulli polynomials. Then, for $t>0$ and $m \in \mathbb{N}_{0}$,

$$
(-1)^{m} \nu_{m}(t)>0 \text {. }
$$

Chen [6, Lemma 1] has proved the statement in (3.1) for $m=0,1,2$, and 3. He has also pointed out in [6] that, if (3.1) is true, then it follows that the function

$$
V_{m}(x)=(-1)^{m}\left\{\ln \frac{\Gamma\left(x+\frac{3}{4}\right)}{x^{1 / 2} \Gamma\left(x+\frac{1}{4}\right)}-\sum_{j=1}^{m} \frac{B_{2 j+1}\left(\frac{1}{4}\right)}{j(2 j+1)} \frac{1}{x^{2 j}}\right\}
$$

for $m \in \mathbb{N}_{0}$ is completely monotonic on $(0, \infty)$.

It was shown in [6] that $\nu_{m}(t)$ can be written as

$$
\nu_{m}(t)=-\frac{1}{2 \cosh \left(\frac{t}{4}\right)}+\sum_{j=0}^{m} \frac{E_{2 j}}{2(2 j) !}\left(\frac{t}{4}\right)^{2 j}
$$

and (3.1) is equivalent to (2.11). Hence, for $t>0$ and $m \in \mathbb{N}_{0}$, it follows that $(-1)^{m} \nu_{m}(t)>0$ holds true.

It was also shown in $[6]$ that

$$
\begin{aligned}
V_{m}(x) & =(-1)^{m}\left\{\int_{0}^{\infty}\left(\frac{e^{t / 4}-e^{3 t / 4}}{e^{t}-1}+\frac{1}{2}\right) \frac{e^{-x t}}{t} \mathrm{~d} t-\sum_{j=1}^{m} \frac{2 B_{2 j+1}\left(\frac{1}{4}\right)}{(2 j+1) !} \int_{0}^{\infty} t^{2 j-1} e^{-x t} \mathrm{~d} t\right\} \\
& =\int_{0}^{\infty}(-1)^{m} \nu_{m}(t) \frac{e^{-x t}}{t} \mathrm{~d} t .
\end{aligned}
$$

We then obtain from (3.3) that for all $m \in \mathbb{N}_{0}$,

$$
(-1)^{n} V_{m}^{(n)}(x)=\int_{0}^{\infty}(-1)^{m} \nu_{m}(t) t^{n-1} e^{-x t} \mathrm{~d} t>0
$$

for $x>0$ and $n \in \mathbb{N}_{0}$. Hence, the function $V_{m}(x)$, defined by (3.2), is completely monotonic on $(0, \infty)$.

\subsection{A double inequality for a ratio of factorials.}

Noting [6, Eq. (3.26)] that $B_{2 n+1}\left(\frac{1}{4}\right)$ can be expressed in terms of the Euler numbers

$$
B_{2 n+1}\left(\frac{1}{4}\right)=-\frac{(2 n+1) E_{2 n}}{4^{2 n+1}} \quad\left(n \in \mathbb{N}_{0}\right),
$$

we find that (3.2) can be written as

$$
V_{m}(x)=(-1)^{m}\left\{\ln \frac{\Gamma\left(x+\frac{3}{4}\right)}{x^{1 / 2} \Gamma\left(x+\frac{1}{4}\right)}+\sum_{j=1}^{m} \frac{E_{2 j}}{j \cdot 4^{2 j+1}} \frac{1}{x^{2 j}}\right\} .
$$

From the inequality $V_{m}(x)>0$ for $x>0$, we then obtain the following 
Corollary 3.1. For $x>0$, we have

$$
x^{1 / 2} \exp \left(-\sum_{j=1}^{2 m} \frac{E_{2 j}}{j \cdot 4^{2 j+1}} \frac{1}{x^{2 j}}\right)<\frac{\Gamma\left(x+\frac{3}{4}\right)}{\Gamma\left(x+\frac{1}{4}\right)}<x^{1 / 2} \exp \left(-\sum_{j=1}^{2 m+1} \frac{E_{2 j}}{j \cdot 4^{2 j+1}} \frac{1}{x^{2 j}}\right) .
$$

The problem of finding new and sharp inequalities for the gamma function $\Gamma(x)$ and, in particular, for the Wallis ratio ${ }^{1}$

$$
\frac{(2 n-1) ! !}{(2 n) ! !}=\frac{1}{\sqrt{\pi}} \frac{\Gamma\left(n+\frac{1}{2}\right)}{\Gamma(n+1)}
$$

has attracted the attention of many researchers (see $[8,9,14,16,17,19]$ and references therein). For example, Chen and Qi [8] proved that for $n \in \mathbb{N}$,

$$
\frac{1}{\sqrt{\pi\left(n+\frac{4}{\pi}-1\right)}} \leq \frac{(2 n-1) ! !}{(2 n) ! !}<\frac{1}{\sqrt{\pi\left(n+\frac{1}{4}\right)}},
$$

where the constants $(4 / \pi)-1$ and $\frac{1}{4}$ are the best possible. This inequality is a consequence of the complete monotonicity on $(0, \infty)$ of the function (see [7])

$$
\Upsilon(x)=\frac{\Gamma(x+1)}{\sqrt{x+\frac{1}{4}} \Gamma\left(x+\frac{1}{2}\right)} .
$$

If we write (3.4) as

$$
\frac{1}{\sqrt{x}} \exp \left(\sum_{j=1}^{2 m+1} \frac{E_{2 j}}{j \cdot 4^{2 j+1}} \frac{1}{x^{2 j}}\right)<\frac{\Gamma\left(x+\frac{1}{4}\right)}{\Gamma\left(x+\frac{3}{4}\right)}<\frac{1}{\sqrt{x}} \exp \left(\sum_{j=1}^{2 m} \frac{E_{2 j}}{j \cdot 4^{2 j+1}} \frac{1}{x^{2 j}}\right)
$$

and replace $x$ by $x+\frac{1}{4}$, we find

$$
\begin{aligned}
\frac{1}{\sqrt{x+\frac{1}{4}}} \exp \left(\sum_{j=1}^{2 m+1} \frac{E_{2 j}}{j \cdot 4^{2 j+1}} \frac{1}{\left(x+\frac{1}{4}\right)^{2 j}}\right)<\frac{\Gamma\left(x+\frac{1}{2}\right)}{\Gamma(x+1)} \\
\quad<\frac{1}{\sqrt{x+\frac{1}{4}}} \exp \left(\sum_{j=1}^{2 m} \frac{E_{2 j}}{j \cdot 4^{2 j+1}} \frac{1}{\left(x+\frac{1}{4}\right)^{2 j}}\right) .
\end{aligned}
$$

Noting that (3.5) holds, we then deduce from (3.6) that

$$
\begin{aligned}
\frac{1}{\sqrt{\pi\left(x+\frac{1}{4}\right)}} \exp \left(\sum_{j=1}^{2 m+1} \frac{E_{2 j}}{j \cdot 4^{2 j+1}} \frac{1}{\left(x+\frac{1}{4}\right)^{2 j}}\right)<\frac{(2 n-1) ! !}{(2 n) ! !} \\
<\frac{1}{\sqrt{\pi\left(x+\frac{1}{4}\right)}} \exp \left(\sum_{j=1}^{2 m} \frac{E_{2 j}}{j \cdot 4^{2 j+1}} \frac{1}{\left(x+\frac{1}{4}\right)^{2 j}}\right),
\end{aligned}
$$

which generalizes a recent result of Chen [6, Eq. (3.40)], who proved the inequality (3.7) for $m=1$.

We remark that Mortici et al. [20] proved that some functions associated with the products $\prod_{k=1}^{n} \frac{3 k-2}{3 k}$ and $\prod_{k=1}^{n} \frac{3 k-1}{3 k}$ are completely monotonic and established some sharp inequalities.

\footnotetext{
${ }^{1}$ Here, we employ the special double factorial notation $(2 n) ! !=2 \cdot 4 \cdot 6 \cdots(2 n)=2^{n} n !, \quad(2 n-1) ! !=1 \cdot 3$. $5 \cdots(2 n-1)=2^{n} \pi^{-1 / 2} \Gamma\left(n+\frac{1}{2}\right)$; see [1, p. 258].
} 


\subsection{Integral representations for $\ln \pi$.}

Sondow and Hadjicostas [31] introduced and studied the generalized-Euler-constant function $\gamma(z)$, defined by

$$
\gamma(z)=\sum_{n=1}^{\infty} z^{n-1}\left(\frac{1}{n}-\ln \frac{n+1}{n}\right)
$$

where the series converges when $|z| \leq 1$. Pilehrood and Pilehrood [24] considered the function $z \gamma(z)(|z| \leq 1)$. The function $\gamma(z)$ generalizes both Euler's constant $\gamma(1)$ and the alternating Euler constant $\gamma(-1)=\ln (4 / \pi)[29,30]$. An interesting comparison by Sondow [29] is the double integral and alternating series

$$
\ln \frac{4}{\pi}=\int_{0}^{1} \int_{0}^{1} \frac{x-1}{(1+x y) \ln (x y)} \mathrm{d} x \mathrm{~d} y=\sum_{n=1}^{\infty}(-1)^{n-1}\left(\frac{1}{n}-\ln \frac{n+1}{n}\right) .
$$

The formula (3.3) can provide integral representations for the constant $\pi$. For example, the choice $(x, m)=(1 / 4,0)$ in $(3.3)$ yields

$$
\int_{0}^{\infty}\left(\frac{e^{t / 4}-e^{3 t / 4}}{e^{t}-1}+\frac{1}{2}\right) \frac{2 e^{-t / 4}}{t} \mathrm{~d} t=\ln \frac{4}{\pi}
$$

which provides a new integral representation for the alternating Euler constant $\gamma(-1)=\ln (4 / \pi)$. The choice $(x, m)=(3 / 4,0)$ in $(3.3)$ yields

$$
\int_{0}^{\infty}\left(\frac{e^{t / 4}-e^{3 t / 4}}{e^{t}-1}+\frac{1}{2}\right) \frac{2 e^{-3 t / 4}}{t} \mathrm{~d} t=\ln \frac{\pi}{3} .
$$

Many formulas exist for the representation of $\pi$, and a collection of these formulas is listed in $[27,28]$. For more history of $\pi$ see $[3,4,10]$.

3.4. The complete monotonicity of a remainder function in the Barnes $G$-function. The following expansion for Barnes $G$-function was established by Ferreira and López [11, Theorem 1]

$$
\begin{aligned}
\ln G(z+1)=\frac{1}{4} z^{2}+z \ln \Gamma(z & +1)-\left(\frac{1}{2} z^{2}+\frac{1}{2} z+\frac{1}{12}\right) \ln z-\ln A \\
& +\sum_{k=1}^{N-1} \frac{B_{2 k+2}}{2 k(2 k+1)(2 k+2) z^{2 k}}+\mathcal{R}_{N}(z) \quad(N \in \mathbb{N})
\end{aligned}
$$

for $|z| \rightarrow \infty$ in $|\arg z|<\pi$, where $B_{2 k+2}$ are the Bernoulli numbers and $A$ is the GlaisherKinkelin constant with the numerical value $1.282427 \ldots$. For $\Re(z)>0$, the remainder $\mathcal{R}_{N}(z)$ is given by

$$
\mathcal{R}_{N}(z)=\int_{0}^{\infty}\left(\frac{t}{e^{t}-1}-\sum_{k=0}^{2 N} \frac{B_{k}}{k !} t^{k}\right) \frac{e^{-z t}}{t^{3}} d t .
$$

Estimates for $\left|\mathcal{R}_{N}(z)\right|$ were also obtained by Ferreira and López [11], showing that the expansion is indeed an asymptotic expansion of $\ln G(z+1)$ in sectors of the complex plane cut along the negative axis. Pedersen [23, Theorem 1.1] proved that for any $N \geq 1$, the function $x \mapsto$ $(-1)^{N} \mathcal{R}_{N}(x)$ is completely monotonic on $(0, \infty)$. 
Here, we present another proof of this complete monotonicity result. From (2.2), we obtain the following inequality:

$$
\sum_{j=0}^{2 m} \frac{2^{2 j} B_{2 j}}{(2 j) !} t^{2 j-1}<\operatorname{coth} t<\sum_{j=0}^{2 m+1} \frac{2^{2 j} B_{2 j}}{(2 j) !} t^{2 j-1} \quad\left(t>0, m \in \mathbb{N}_{0}\right),
$$

which is equivalent to

$$
(-1)^{N}\left(\operatorname{coth} t-\sum_{j=0}^{N} \frac{2^{2 j} B_{2 j}}{(2 j) !} t^{2 j-1}\right)>0 \quad\left(t>0, N \in \mathbb{N}_{0}\right) .
$$

Replacement of $t$ by $t / 2$ in the above inequality yields

$$
(-1)^{N}\left(\frac{t}{2} \operatorname{coth}\left(\frac{t}{2}\right)-\sum_{j=0}^{N} \frac{B_{2 j}}{(2 j) !} t^{2 j}\right)>0 \quad\left(t>0, N \in \mathbb{N}_{0}\right) .
$$

Accordingly, we obtain from (3.9) that the function

$$
(-1)^{N} \mathcal{R}_{N}(x)=\int_{0}^{\infty}(-1)^{N}\left(\frac{t}{2} \operatorname{coth}\left(\frac{t}{2}\right)-\sum_{k=0}^{N} \frac{B_{2 k}}{(2 k) !} t^{2 k}\right) \frac{e^{-x t}}{t^{3}} d t
$$

is completely monotonic on $(0, \infty)$.

\section{A quadratic recurrence relation for $B_{n}$}

Euler (see [22, p. 595, Eq. (24.14.2)] and [37]) presented a quadratic recurrence relation for the Bernoulli numbers:

$$
\sum_{k=0}^{n}\left(\begin{array}{l}
n \\
k
\end{array}\right) B_{k} B_{n-k}=(1-n) B_{n}-n B_{n-1} \quad(n \geq 1),
$$

which is equivalent to ${ }^{2}$

$$
\sum_{j=1}^{n-1}\left(\begin{array}{l}
2 n \\
2 j
\end{array}\right) B_{2 j} B_{2 n-2 j}=-(2 n+1) B_{2 n} \quad(n \geq 2) .
$$

Other quadratic recurrences for the Bernoulli numbers have been given by Gosper (see [35, Eq. $(38)])$ as

$$
B_{n}=\frac{1}{1-n} \sum_{k=0}^{n}\left(1-2^{1-k}\right)\left(1-2^{k-n+1}\right)\left(\begin{array}{l}
n \\
k
\end{array}\right) B_{k} B_{n-k}
$$

and by Matiyasevitch [18] (see also [37]) as

$$
B_{n}=\frac{1}{n(n+1)} \sum_{k=2}^{n-2}\left\{n+2-2\left(\begin{array}{c}
n+2 \\
k
\end{array}\right)\right\} B_{k} B_{n-k} \quad(n \geq 4) .
$$

Here, we present a (presumably new) quadratic recurrence relation for the Bernoulli numbers.

\footnotetext{
${ }^{2}$ The relation (4.1) can be used to show by induction that $(-1)^{n-1} B_{2 n}>0$ for all $n \geq 1$; that is, the even-index Bernoulli numbers have alternating signs.
} 
Theorem 4.1. The Bernoulli numbers satisfy the following quadratic recurrence relation:

$$
B_{n}=\frac{1}{2^{n}-1} \sum_{k=2}^{n-2}\left(1-2^{k}\right)\left(\begin{array}{l}
n \\
k
\end{array}\right) B_{k} B_{n-k} \quad(n \geq 4) .
$$

Proof. If we replace $t$ by $t / 2$ in (2.1), we find

$$
\frac{2}{e^{t / 2}+1}=1+\sum_{j=2}^{\infty} b_{j} t^{j-1}, \quad b_{j}=\frac{2\left(1-2^{j}\right) B_{j}}{2^{j-1} \cdot j !} .
$$

The Bernoulli numbers $B_{n}$ are defined by the generating function

$$
\frac{t}{e^{t}-1}=\sum_{n=0}^{\infty} B_{n} \frac{t^{n}}{n !}
$$

which yields

$$
\frac{t / 2}{e^{t / 2}-1}=\sum_{k=0}^{\infty} \frac{B_{k} t^{k}}{2^{k} k !}
$$

It then follows from (4.3) and (4.5) that

$$
\begin{aligned}
\frac{t}{e^{t}-1} & =\left(1+\sum_{j=2}^{\infty} b_{j} t^{j-1}\right) \sum_{k=0}^{\infty} \frac{B_{k} t^{k}}{2^{k} k !} \\
& =\sum_{k=0}^{\infty} \frac{B_{k} t^{k}}{2^{k} k !}+\sum_{j=2}^{\infty} b_{j} t^{j-1} \sum_{k=0}^{\infty} \frac{B_{k} t^{k}}{2^{k} k !} \\
& =\sum_{j=0}^{\infty} \frac{B_{j} t^{j}}{2^{j} j !}+\sum_{j=1}^{\infty} \sum_{k=0}^{j-1} b_{k+2} \frac{B_{j-k-1} t^{k}}{2^{j-k-1}(j-k-1) !},
\end{aligned}
$$

that is

$$
\frac{t}{e^{t}-1}=\sum_{j=0}^{\infty}\left(\frac{B_{j}}{2^{j} j !}+\sum_{k=0}^{j-1} b_{k+2} \frac{B_{j-k-1}}{2^{j-k-1}(j-k-1) !}\right) t^{j} .
$$

Equating coefficients of equal powers of $t$ in (4.4) and (4.6), we see that

$$
\frac{B_{j}}{j !}=\frac{B_{j}}{2^{j} \cdot j !}+\sum_{k=0}^{j-1} b_{k+2} \frac{B_{j-k-1}}{2^{j-k-1} \cdot(j-k-1) !} \quad\left(j \in \mathbb{N}_{0}\right) .
$$

Substitution of the coefficients $b_{j}$ in (4.3) into (4.7) then yields

$$
B_{j}=\frac{j !}{2^{j}-1} \sum_{k=0}^{j-1} \frac{2\left(1-2^{k+2}\right) B_{k+2} B_{j-k-1}}{(k+2) ! \cdot(j-k-1) !} \quad(j \in \mathbb{N}) .
$$

It is easy to see that

$$
\begin{aligned}
B_{j} & =\frac{j !}{2^{j}-1}\left(\sum_{k=0}^{j-3} \frac{2\left(1-2^{k+2}\right) B_{k+2} B_{j-k-1}}{(k+2) ! \cdot(j-k-1) !}-\frac{\left(1-2^{j}\right) B_{j}}{j !}+\frac{2\left(1-2^{j+1}\right) B_{j+1}}{(j+1) !}\right) \\
& =\frac{j !}{2^{j}-1} \sum_{k=0}^{j-3} \frac{2\left(1-2^{k+2}\right) B_{k+2} B_{j-k-1}}{(k+2) ! \cdot(j-k-1) !}+B_{j}+\frac{2\left(1-2^{j+1}\right) B_{j+1}}{\left(2^{j}-1\right)(j+1)} .
\end{aligned}
$$


We therefore obtain

$$
B_{j+1}=\frac{1}{2^{j+1}-1} \sum_{k=0}^{j-3}\left(1-2^{k+2}\right)\left(\begin{array}{l}
j+1 \\
k+2
\end{array}\right) B_{k+2} B_{j-k-1} \quad(n \in \mathbb{N} \backslash\{1,2\}),
$$

which, upon replacing $j$ by $n-1$ and $k$ by $k-2$, yields (4.2). This completes the proof of Theorem 4.1.

\section{REFERENCES}

[1] M. Abramowitz, I.A. Stegun (Editors), Handbook of Mathematical Functions with Formulas, Graphs, and Mathematical Tables, Applied Mathematics Series 55, Ninth printing, National Bureau of Standards, Washington, D.C., 1972.

[2] H. Alzer, On some inequalities for the gamma and psi functions, Math. Comp. 66 (1997) 373-389.

[3] P. Beckmann, A History of Pi, St. Martin's Press, New York, New York, 1971.

[4] L. Berggren, J. Borwein, P. Borwein, eds., Pi: A Source Book, 2nd ed., Springer, New York, 2000.

[5] B.C. Berndt, Ramanujan's Notebooks, Part V. Springer, Berlin, 1998.

[6] C.-P. Chen, Inequalities and completely monotonic functions associated with the ratios of functions resulting from the gamma function, Appl. Math. Comput. 259 (2015) 790-799.

[7] C.-P. Chen, F. Qi, Completely monotonic function associated with the gamma functions and proof of Wallis' inequality, Tamkang J. Math. 36 (2005) 303-307.

[8] C.-P. Chen, F. Qi, The best bounds in Wallis' inequality, Proc. Amer. Math. Soc. 133 (2005) 397-401.

[9] C.-P. Chen, R.B. Paris, Inequalities, asymptotic expansions and completely monotonic functions related to the gamma function, Appl. Math. Comput. 250 (2015) 514-529.

[10] W. Dunham, Journey Through Genius, The Great Theorems of Mathematics, Penguin, 1990.

[11] C. Ferreira, J.L. López, An asymptotic expansion of the double gamma function, J. Approx. Theory 111 (2001) 298-314.

[12] S. Koumandos, Remarks on some completely monotonic functions, J. Math. Anal. Appl. 324 (2006) 14581461.

[13] S. Koumandos, H.L. Pedersen, Completely monotonic functions of positive order and asymptotic expansions of the logarithm of Barnes double gamma function and Euler's gamma function, J. Math. Anal. Appl. 355 (2009) 33-40.

[14] S. Koumandos, Remarks on a paper by Chao-Ping Chen and Feng Qi, Proc. Amer. Math. Soc. 134 (2006) $1365-1367$.

[15] S. Koumandos, On completely monotonic and related functions, Mathematics without Boundaries, 285-321, Springer, New York, 2014.

[16] V. Lampret, Wallis sequence estimated through the Euler-Maclaurin formula: even from the Wallis product $\pi$ could be computed fairly accurately, Austral. Math. Soc. Gaz. 31 (2004) 328-339.

[17] V. Lampret, An asymptotic approximation of Wallis' sequence, Cent. Eur. J. of Math. 10 (2012) 775-787.

[18] Y. Matiyasevich, Identities with Bernoulli numbers, Identity \#0202, 1997. Available at: http://logic.pdmi. ras.ru/ yumat/Journal/Bernoulli/bernoulli.htm. Accessed 23 Apr 2015

[19] C. Mortici, New approximation formulas for evaluating the ratio of gamma functions, Math. Comput. Modelling 52 (2010) 425-433.

[20] C. Mortici, V.G. Cristea, D. Lu, Completely monotonic functions and inequalities associated to some ratio of gamma function. Appl. Math. Comput. 240 (2014) 168-174.

[21] M.E. Muldoon, Some monotonicity properties and characterizations of the gamma function, Aequationes Math. 18 (1978) 54-63.

[22] F.W.J. Olver, D.W. Lozier, R.F. Boisvert, C.W. Clarks (eds.), NIST Handbook of Mathematical Functions, Cambridge University Press, New York, 2010.

[23] H.L. Pedersen, On the remainder in an asymptotic expansion of the double gamma function, Mediterr. J. Math. 2 (2005) 171-178.

[24] K.H. Pilehrood, T.H. Pilehrood, Arithmetical properties of some series with logarithmic coefficients, Math. Z. 255 (2007) 117-131.

[25] G. Pólya, G. Szegö, Problems and Theorems in Analysis, Vol. I and II, Springer-Verlag, Berlin, Heidelberg, 1972.

[26] Z. Sasvári, Inequalities for binomial coefficients. J. Math. Anal. Appl. 236 (1999) 223-226. 
[27] A. Sofo, Some representations of $\pi$, Austral. Math. Soc. Gaz. 31 (2004) 184-189.

[28] A. Sofo, $\pi$ and some other constants, J. Inequal. Pure Appl. Math. 6 (2005), no. 5, Article 138. (electronic).

[29] J. Sondow, Double integrals for Euler's constant and $\ln (4 / \pi)$ and an analog of Hadjicostas's formula, Amer. Math. Monthly, 112 (2005) 61-65.

[30] J. Sondow, New Vacca-type rational series for Euler's constant and its alternating analog $\ln (4 / \pi)$, Additive Number Theory, Festschrift in Honor of the Sixtieth Birthday of M.B. Nathanson (D.Chudnovsky and G. Chudnovsky, eds.), Springer, 2010. arXiv:0508042[math.NT]

[31] J. Sondow and P. Hadjicostas, The generalized-Euler-constant function $\gamma(z)$ and a generalization of Somos's quadratic recurrence constant, J. Math. Anal. Appl. 332 (2007) 292-314.

[32] H.M. Srivastava, J. Choi, Series associated with the zeta and related functions, Kluwer Academic Publishers, Dordrecht, Boston and London, 2001.

[33] N.M. Temme, Special Functions: An introduction to the classical functions of mathematical physics, Wiley, 1996.

[34] Z.X. Wang and D.R. Guo, Special Functions, World Scientific, Singapore, 1989.

[35] E.W. Weisstein, "Bernoulli Numbers." From MathWorld-A Wolfram Web Resource. Available at http: //mathworld.wolfram.com/BernoulliNumber.html. Accessed 10 Apr 2015

[36] E.T. Whittaker, G.N. Watson, A Course of Modern Analysis, 4th ed., Cambridge University Press, Cambridge, 1966.

[37] Wikipedia contributors, Bernoulli numbers, Wikipedia, the free encyclopedia. Available at https://en. wikipedia.org/wiki/Bernoullinumbers. Accessed 10 Apr 2015

[38] Y. Xu, X. Han, Complete monotonicity properties for the gamma function and Barnes $G$-function, Sci. Magna 5 (2009) 47-51.

C.-P. Chen: School of Mathematics and Informatics, Henan Polytechnic University, Jiaozuo City 454000, Henan Province, China

E-mail address: chenchaoping@sohu.com

R.B. Paris: Division of Computing and Mathematics, University of Abertay, Dundee, DD1 1HG, UK E-mail address: R.Paris@abertay.ac.uk 